\title{
A Review on Resource Allocation Techniques in Small Cell LTE-A Heterogeneous Networks
}

\author{
Amandeep ${ }^{1}$, Sanjeev Kumar ${ }^{2}$ \\ Assistant Professor, Department of CSE, Guru Jambheshwar University of Science \& Technology, Hisar, India ${ }^{1,2}$
}

\begin{abstract}
According to originating standards for LTE-A mobile networks, the small cells are expected to be deployed in future mobile and wireless networks to improve coverage in specific areas with low signal quality. By placing additional stations to the network, new cell boundaries are introduced. Since heavy deployment of the small cells with low radius is expected in LTE-A networks, the procedures for the user's resource allocation becomes more frequently. Therefore, more often scanning of higher amount of entities in UE's neighborhood must be performed. Moreover, each resource allocation generates some management overhead and introduces interruption in user's communication. All these aspects lead to a drop of user's throughput and QoS. This is getting more apparent with dense deployment of small cells. Hence, large and efficient deployment of the small cells requires optimizing the principles of user's service allocation support to ensure continuous high level of service quality.
\end{abstract}

Keywords: LTE-A mobile networks, QoS, Wireless Networks, Heterogeneous Networks, E- UTRAN.

\section{INTRODUCTION}

The growth in the mobile subscriber will be further intensified by adaptation of broadband mobile access technologies in densely populated and developing countries such as India, etc. Mobile multimedia traffic is increasing more rapidly than voice and will increasingly traffic flow. The technology shift from circuit switched air interface design to full IP-based has provided user with the ability to more efficiently, more reliably, and more securely utilized multimedia services including file transfer, social network, video streaming, web browsing, cloud synchronization/ cloud web-based applications, navigation, online gaming, voice over IP (VoIP) and location based multicast and broadcast services. These services can be uplink/downlink and real time and nonreal time with different QoS requirements. The new application consume large bandwidths, cause of the requirements of higher data rate and throughputs.

Improved quality and increasing capacity of the wireless network have become a priority for the standard development organization such as ITU, IMT and 3GPP. The 3rd Generation Partnership Project (3GPP) is a collaboration between seven groups of telecommunication associations (ARIB, ATIS, CCSA, ETSI, TSDSI, TTA,
TTC), which are known as the Organizational Partner [1]. $3 \mathrm{GPP}$ is an organization that working on the cellular network technology, i.e. radio access technique, core network, services capabilities, security and quality of services. They provide stable technical basis to transpose into their own specification. There are four technical specification groups in 3GPP which include RAN, Service and system aspect (SA), Core network and terminals (CT), and GSM EDGE radio access network (GERAN). Main motive of the organization is to provide a stable environment to its users with better Quality of service, security fast access and handover/interface between the previous technologies.

3GPP initiated a project on the long term evolution (LTE) in 2004 to meet the requirements of the user on UMTS (Universal mobile telecommunication system) radio access network (RAN). They proposed E- UTRAN (Evolved UMTS terrestrial radio access network) improved end user throughput, system capacity, reduced user-plane and control plane latencies [2]. The main objectives of LTE were to minimize the system and user equipment complexities [3].

Table 1. Mobile Generations

\begin{tabular}{|c|l|l|l|l|}
\hline Generation & Period & Transmission & Services & Examples \\
\hline 1G & 1980 's & Analog FM ,FDMA / FDD & Voice & AMPS \\
\hline 2G & 1990 's & $\begin{array}{l}\text { Digital modulation } \\
\text { TDMA/CDMA }\end{array}$ & Voice, SMS & $\begin{array}{l}\text { GSM,IS-95, GPRS, } \\
\text { EDGE }\end{array}$ \\
\hline 3G & $2000+$ & Wideband modulation & $\begin{array}{l}\text { Internet, email, Multi-media } \\
\text { streaming, etc. }\end{array}$ & $\begin{array}{l}\text { WCDMA, } \\
\text { HSDPA, HSUPA }\end{array}$ \\
\hline 4G & $2005+$ & OFDMA & Mobile internet, mobile video etc & LTE, WiMAX \\
\hline
\end{tabular}




\section{LTE-Advanced}

Enhancement in LTE system by 3GPP is continuously in the form of releases, LTE-Advanced described in R-10 and R-11 major evolution in the spectral efficiency and bandwidth (100Mhs bandwidth and 1Gbps peak throughputs). Some technologies (i.e.) carrier aggregation, coordinate multipoint scheduling, SU-MIMO/ MUMIMO, relay node, small cell enhanced inter-cell interference coordination and enhanced multimedia broadcast multicast services were introduced to achieved these targets [78].

Available spectrum for mobile communication is limited and every growing mobile broadband traffic demands cannot be served by the existing LTE macro network only [52]. LTE small cell deployment is thus seen an essential mean to provide the high capacity for very densely populated outdoor and indoor areas. Network densification using small cell network has been an important evolution in future generation network [20].

\section{Small Cell Overview}

The idea of small cell originated around 1984 [17] in which macro cell radius is divided into small radius or small parts called small cell. The reason behind small cell deployment is capacity and the improvement of user throughput. Simultaneously the idea was introduced by mobile network operators facing the problems of signal quality and poor coverage regions in indoor areas. In 1990's pico cell was introduced [18] to fulfill the gap of capacity and coverage problem in overloaded macro cell. The needs of the small cell [33]

1. Number of mobile user increase and base station increases.

2. Base station move closer to user.

3. Energy and resource cost rise.

Small cells forum, was a collaborative operation of different organizations in 2007 [19], objective of developing open standards for product interoperability. Small Cell Forum [24] named small cells as "low-power wireless access points that operate in licensed spectrum, are operator-managed and feature edge-based intelligence. They provide improved cellular coverage, capacity and applications for homes and enterprises as well as metropolitan and rural public spaces [79]. Small cell include technologies variously described as femtocells, picocells, metro cells and microcells [broadly increase the size from femto cells (smallest) to micro cells (largest)]." The combination of these networks are called heterogonous network [33]. Cell range of the traditional cell is tens to hundred meters. Small cell deployment [26] in residential area to remove the coverage problem called femto-cells (small cell). Small cells are well suited to deployment in rural villages, remote industrial sites, on transportation, as well as temporary networks for public safety and special events [25].

3GPP initiated a workshop [27] on further steps in the evolution of LTE toward the future. There were 42 presentations from 3GPP member organizations, including network operators considering future requirements and candidate technologies. The key areas of enhancement that were identified included capacity increase to cope with the traffic explosion, energy savings, cost efficiency, support for diverse application and traffic types, higher user experience/data rate, and backhaul enhancement. As a potential technology to meet these requirements, a great majority of companies showed interest in enhanced small cells. Small cell have become a hot topic for research as evidenced by a significantly increases publication in this area, 3 in 2007 to 10 (2008), 51 (2009), 116 (2010), are registered in IEEE data based and continuing increased. However small cell is increased, there number of problems and challenged were also increased. Some major challenges are listed in below, and few of them will be described in literature to take a problem in this paper.

- Cell selection

- Mobility and interference issue

- Advanced technology such as enhanced inter-cellinterference coordination (eICIC)

- Inter-site carrier aggression

- Coordinate multipoint transmission (CoMP)

- Enhanced interference management and traffic adaption (eIMTA)

- Dual connectivity also depend on the architecture and the level of coordination between macro and small cell

- Self organization and self optimization

- Radio resource management and central control plane termination using dual connectivity

- For dense and ultra-dense small cell deployment with internet large traffic variations

- On/off switching become more important for energy saving and interference reduction.

\section{SMALL CELL CHALLENGES AND RELATED LITERATURE REVIEW}

After a brief introduction about LTE/LTE-A and small cell, now we are going to discuss about challenges in small cell/Heterogeneous networks deployment. Small cell are deployed on the same or different frequency layer as the macro network, which will result in different challenges in term of [28][79]:

\section{Intercell Interference}

Intercell interference is the most significantly and widelydiscussed challenged in small cell deployment. That is arising between the small cell (femto, pico and heterogeneous network cell) and macro cells. We are going to discuss the most commonly used femto small cell. Femto cell is control by HeNB that was described by 3GPP release 8/9/10 [25]. HeNB also called Femto Access Point (FAP). Major cause of interference between FAP and macro cell is, low power FAP is deployed in the cell range of macro cell. Two type interference described in literature [28][33][13], intercell (cross layer) and intra-cell (co layer). Intercell interference between the FAP and macro cell while intra cell interference is between two 
femto access points. Cross-tier and intra-tier interference problems are significantly challenging due to backhaul, restricted access control and self organizing nature described by, David Lopez-Perez et al. [52]. To overcome the effect of interference, cancellation techniques have been proposed by S.P. Wiber et al. [40]. But this approach was discarded due to errors in the cancellation process. The simplest forms of inter-cell interference mitigation are Fractional frequency reuse (FFR) schemes because it is easy to implement in OFDA system such as LTE. Although these techniques do reduce the interference by partitioning the overall bandwidth among cells impairs the overall throughput. FFR used the static power allocation that is not applicable for dynamic nature of femto cells. In addition this, Poongup Lee et al. [41] proposed an interference management scheme in the LTE femtocell systems using Fractional Frequency Reuse (FFR), according to this approach frequency band is allocating Under the macro cell, the femtocell chooses sub-bands which are not used in the macro cell sub-area to avoid interference. This proposed scheme enhanced total/edge throughputs and reduces the outage probability in overall network, especially for the cell edge users. Enhanced frequency reuse schemes, such as Partial Frequency Reuse (PFR) proposed by Sternad M. et al. [42] and Soft Frequency Reuse (SFR) [43], have thus been introduced. The idea behind PFR is to partition the bandwidth so that only a limited amount of RBs can be used by all cells, while others are used with higher reuse factor. Cell-edge UEs can take advantage of lower interference in these sub-bands. Enhancement in PFR presented by Bujar Krasniqi et al. [44] a Flexible Bandwidth Allocation (FBA) scheme for Partial Frequency Reuse (PFR) depending on the network-load, which allocates bandwidth dynamically in the network. That presents general framework for intelligent frequency planning in wireless networks. In the SFR scheme, a cell can allocate the entire sub frame, but different power levels are employed in cell-center and cell-edge RBs. Semi-static Inter-Cell Interference Coordination (ICIC) schemes were then proposed, based on the above frequency reuse schemes. Fang L. et al. [45] and Yiwei Yu et al. [46] authors proposed a novel load distribution aware soft frequency reuse (LDA-SFR) scheme for intercell interference mitigation and performance optimization in next generation wireless networks. The proposed scheme consists of two novel algorithms: edge bandwidth reuse and centre bandwidth compensation. Using the edge bandwidth reuse algorithm, cell-edge users can take advantage of uneven traffic load and user distributions within each cell to expand their resource allocations. Studied by Ebrahim Baktash et al. [47] dynamic intracell subcarrier reuse in cooperative OFDMA networks in which the users are allowed to share any subcarrier in the relay links provided that the resultant intra-cell interference is managed. Reham Almesaeed et al. [48] compared the downlink throughput macro-cell interference scenario with a frequency reuse scheme. Bit accurate link level simulations are performed for the downlink physical shared channel (PDSCH). In addition, A Ghosh et al. [49] and A. Damnjanovic et al. [50] discussed the advanced methods for intercell interference coordination (ICIC) specifically for femtocell network, it has been a major motivation for the 3GPP LTE-Advanced standardization effort. A new inter-cell interference coordination (ICIC) scheme based on adaptive sub-band avoidance on the inter-cell level is proposed by Han Xiao et al. [51] where for each cell a certain ratio of the subcarriers are avoided in the subcarriers group used for the center region. David Lopez-Perez et al. [52] Particular attention has been given to the avoidance of cross-tier interference due to its crucial role in proper operation of multi-tier networks. Furthermore, the main focused was on eICIC techniques. More advanced techniques for interference control including backhaul-based [77], and cooperative communication between multiple base stations are also being researched G. Boudreau et al. [53] and S. Rangan et al. [54]. These all we will be discuss in resource allocation challenged.

\section{Resource Allocation}

The minimum time -frequency resource unit used for downlink transmission is resource element, which is defined as one sub carrier over one OFDM symbol. For both TDD and FDD duplex scheme, a group of 12 subcarrier continuous in frequency over one slot in time from a PRB (physical resource block) a two dimension region corresponding to one slot in the time-domain and 180 KHZ $(12 * 15 \mathrm{KHZ})$ in the frequency-domain. The transmissions are allocated in units of PRB. More specifically, the data or control signaling in each slot is transmitted via one or several resource elements of sub carrier over OFDM symbols. Small cell is most provisioning idea to improve the spectral efficiency, reduced cost and enhancement of the throughput. Resource allocation method is very important to improve such objectives. A lot recently research have been done in this area Parag KulKarni et al. [54] described two possible deployment path for operator, one approach is to set aside a chunk of the spectrum for deploying femto cells and use the remaining for the macro network. This is well known as the dedicated channel deployment approach. The second approached deploy femtocells on the same spectrum as existing macro cells. This is well known as co-channel deployment approach. Abbas Hatoum et al. [55] related works is the computation of efficient allocation of time-frequency resource blocks, while accounting for cross layer interference (interference between macro cell and femtocells) and co-layer interference (interference between femtocells). Yu-jung chang et al. [56] proposed graph theory to managed the inter cell interference coordination in multi-cell OFDMA downlink resource allocation. In the collaborative resource allocation scheme K Lee et al. [57], cross-tier interference is approximated as additive white Gaussian noise (AWGN) for self healing and self optimization network. In the Lagrangian dual decomposition-based resource allocation scheme J Zhang et al. [58], constraints on 
cross-tier interference are used in power allocation, but channels are assigned randomly to femto users. Authors K. Sundaresan et al. [59] proposed a location-based resource management scheme to achieve efficient resource allocation and spatial reuse. In contrast $\mathbf{V}$. this:

Chandrasekhar et al. [60] proposed a decentralized resource allocation scheme that guarantees users a prescribed quality of service. A. Valcarce et al. [61] Enhanced and proposed a hybrid limited access method that reduces cross-layer interference while guaranteeing subscribers a minimum performance. N. Arulselvan et al. [62], S. G. Quan et al. [63] and G. He et al. [64] described some works applying game theory and selfish characteristics in femtocell networks were focused on signal-to-interference-ratio-based power control resource allocation.

Besides, 3GPP introduced the advancement in the form of LTE-Advance, which is backward capability with LTE and its previous technology. In LTE-A major focused to achieve higher data rate and maximum throughput using the different technique such as carrier aggression, enhanced intercell interference coordination and coordinate multipoint. These technologies create the road map for $5 \mathrm{G}$ network and increase the demands of heterogeneous networks. These techniques used as the resource allocation in networks. As we earlier discuss about resources or physical resource block. Here first we present some basic information about that new advancement in LTE network and after that literature about this.

\section{Carrier Aggression}

Introduced by $3 \mathrm{GPP}$ in release $8 / 9$ [21], to increase the bandwidth of network using carrier aggression in FDD and TDD frame called carrier component. Each component can have a bandwidth of $1.4,3,5,10,15$ or $20 \mathrm{MHz}$. Maximum five components can be aggregated at a time, if $20 \mathrm{MHz}$ component aggregate one time the maximum bandwidth will be $100 \mathrm{MHz}$. carrier aggression in either both DL/UL side or DL side, but not in only UL side, and DL carrier always be greater than UL carrier component [32]. Each UE has a single serving cell that provides all necessary control information and function. The serving cell is known as the primary cell and other component carrier that help the primary carrier component is called the secondary cell [7]. Carrier aggression component classified into three categories according to their resource allocation techniques [30]:

Intra-band contiguous carrier aggression: It is the simplest form of carrier aggression this type of carrier aggression uses a single frequency band.

Intra-band non-contiguous carrier aggression: In this carrier aggression technique aggregated carrier are not adjacent and the multi-carrier cannot treated as a single. The entire carriers are in single frequency band.

Inter-band non-contiguous carrier aggression: In this approach all carrier are in different carrier frequency band.
Enhanced Intercell Interference Coordinates (eICIC)

In this resource allocation method, resource is allocated to improve the interference between the microcell user and small cell user [76]. Two major techniques are used for - Almost Blank Sub frames and

- Reduced power almost blank symbol.

One more technique used [31] Further enhancement in intercell interference coordination for transmitter and receiver based called FeICIC.

\section{Coordination Multipoint (CoMP)}

CoMP (Coordinated Multi-Point Transmission/reception) was introduced in Rel.11, where geographically separated nodes interwork together in transmitting/receiving signals. Three kinds of schemes are defined in downlink [80]:

Joint transmission (JT), In JT, the data toward a single user are simultaneously transmitted from multiple nodes using the same radio resource, which can avoid co-channel interference and increase diversity effect especially for cell edge users.

Dynamic point selection (DPS), in this approach data from network are transferred also to neighboring cells like JT, but only one cell is selected for transmission according to the channel status. By muting on the same radio resource at the neighboring cells (dynamic point blanking: DPB), it can also mitigate the co-channel interference.

Coordinated scheduling (CS), In CS, data from network are transferred to only one node, so independent data are stored in different nodes. If coordinated beam forming (CB) is applied for each node, different data are transmitted simultaneously on the same radio resource without interference. [29]. A short literature is described in below about in these LTE-A technologies.

S. Xu et al. [77] proposed an enhanced ICIC mechanism to suppress such interference by blanking two OFDM symbols. Furthermore, for Release 10 victim users, a novel repetitively designing PDCCH scheme proposed such that the interference can be avoided effectively. M. A. L. Sarker et al. [73] describe the performance of CoMP and estimates an achievable angular channel model for LTE-A using minimum mean square error (MMSE) receiver which efficiently improves the bit-error-rate (BER) and cell-edge throughput per user. A. Davydov et al. [74] investigate the Joint Transmission (JT) CoMP performance in a C-RAN implementation of LTE-A HetNet with large CoMP cluster sizes. Q. Wang et al. [75] analysis the time division duplex (TDD) LTE-A, (i.e.) TD-LTE-A, the channel reciprocity property could be exploited for downlink channel state information (CSI) estimation through the uplink signal, which makes the channel pre-coding more precise compared to the limited codebook feedback. Y. Wang et al. [76] distributed CoMP method for CRE with ABS is proposed in heterogeneous Networks. 


\section{International Journal of Advanced Research in Computer and Communication Engineering} ISO 3297:2007 Certified

Vol. 5, Issue 12, December 2016

\section{CONCLUSION}

After study it has been concluded that demand of data traffic is increased broadly. Hence the spectrum required for each demand is necessary and challenging part for densification of the network. Macro cell is not satisfying the requirement of indoor area. So small cell play the major role to developing the road map of the future generation or $5 \mathrm{G}$ and improved the spectrum and coverage problem in indoor and densification network. However, resource allocation takes an important part for deployments the small cell or Het-Net in the macro cell regions. Our main purpose of study to identify the different resource allocation method that is responsible for improved the cell capacity, over all throughputs, reliability of the LTE-A network and create a roadmap for $5 \mathrm{G}$ technology.

\section{REFERENCES}

[1] http://www.3gpp.org.

[2] 3GPP TS 36.300: "Evolved Universal Terrestrial Radio Access (EUTRA) and Evolved Universal Terrestrial Radio Access Network (E-UTRAN); Over all description; Stage 2".

[3] 3GPP TS 36.401: "Evolved Universal Terrestrial Radio Access Network (E-UTRAN); Architecture description".

[4] L. Ghedia, "Developments in mobile satellite communications", IEEE Workshop on Microwave and Millimetre-Wave Communications - the Wireless Revolution, pp. 1-4, 29, Nov. 1995.

[5] R. M. Buehrer, "Code Division Multiple Access (CDMA)", Morgan \& Claypool, IEEE Synthesis Lectures on Communications, Vol. 1, No. 1, pp.1-192, 2006

[6] 3GPP TSG RAN T RP 30.375: "Overview of 3GPP Release 5 Summary and Features", Version 0.10, June 2003.

[7] Sassan Ahmadi, "LTE-Advanced A Practical System Approach to Understanding 3GPP LTE Releases 10 and 11 Radio access Technologies", First ed., ELSEVIER Academic Press, 2014.

[8] White paper, "EDGE, HSPA and LTE - The Mobile Broadband Advantage", published by Rysavy Research, September 2007.

[9] E. Dahlman, S. Parkvall, J. Skold, and P. Beming, "3G Evolution HSPA and LTE for Mobile Broadband", Academic Press, 2nd edition, 2008

[10] White Paper, "Long Term Evolution Protocol Overview", published by freescale semiconductor, 2008.

[11] Stefan Schwarz, and Markus Rupp, "Evaluation of Distributed Multi-User MIMO-OFDM With Limited Feedback", IEEE Transactions On Wireless Communications, Vol. 13, No. 11, pp. 6081-6094, November 2014.

[12] Chaiman Lim, Taesang Yoo, Bruno Clerckx, Byungju Lee and Byonghyo Shim, "Recent Trend of Multiuser MIMO in LTEAdvanced", IEEE Communications Magazine, vol.51, Issue 3, pp. 127-135, 2013.

[13] White Paper, "Small cell Big Oppotinuies", by Dr. Yan Q Bian, Deepak Rao, published by A Huawei, Feb. 2014.

[14] Astely, D.,Dahlman, E., Furuskar, A., Jading., Lindstrom, M. and Parkvall, S., "LTE: The Evolution of Mobile Broadband", IEEE Communications Magazine, Vol. 47, pp. 44-51, 2009.

[15] Akyildiz, I., Estevez, D. and Reyes E., "The Evolution to $4 \mathrm{G}$ Cellular system: LTE Advanced", Elsevier Physical Communication, Vol. 3, No. 03, pp. 217-244, 2010.

[16] White Paper "The LTE Architecture", published by Alcatel Lucent.

[17] C. stocker, "Small-Cell Mobile Phone Systems", IEEE Transactions on Vehicular Technology, Vol.-33, No. 4, November 1984

[18] Raju lyer, Jerry Parker, Prem Sood, "Intelligent Networking For Digital Cellular Systems And The Wireless World", IEEE Global Telecommunications Conference and Exhibition, 'Communications: Connecting the Future', GLOBECOM '90', Vol. 1, pp. 475-479, 1990.

[19] http://www.smallcellforum.org/
[20] 3GPP TS 36.913: "Requirements for Further Advancements for Evolved Universal Terrestrial Radio Access (E-UTRA) (LTEAdvanced), Release 11", September 2012.

[21] 3rd Generation Partnership Project: "3GPP - Releases, www.3gpp.org/releases", (2013).

[22] 3GPP TR 25.882: "3GPP system Architecture Evolution Report on Technical options and conclusions (Release 8)", sept.2008.

[23] 3GPP TR 25.820: "3G Home NodeB Study Item Technical Report, (Release 8)", 2008.

[24] Small cell forum: "Crossing the Chasm: Small Cells Industry", November 2015.

[25] Small cell forum: DOCUMENT 105.06.01,"Rural and remote: Overview", February 2015.

[26] I. Ashraf, F. Boccardi, L. Ho, "SLEEP mode techniques for small cell deployments", IEEE communication Magazine, Vol. 49, Issue 8, pp. 72-79, 2011.

[27] 3GPP, RWS-120045, "Summary of 3GPP TSG-RAN Workshop on Release 12 and Onward", June 2012.

[28] Jeffrey G. Andrews, Holger Claussen, Mischa Dohler, Sundeep Rangan, Mark C. Reed, "Femtocells: Past, Present, and Future", IEEE Journal On Selected Areas In Communications, Vol. 30, NO. 3, pp. 497-507, APRIL 2012.

[29] Jing Wang, Satoshi Nagata, Lan Chen, Huiling Jiang, "Low Complexity Centralized Scheduling Scheme for Downlink CoMP", IEEE Transactions on Communications, Vol. E98-B No.5, pp. 940948, may 2015.

[30] Klaus Ingemann Pedersen, Frank Frederiksen, Claudio Rosa,Hung Nguyen, Luis Guilherme Uzeda Garcia, and Yuanye Wang, "Carrier Aggregation for LTE-Advanced: Functionality and Performance Aspects", IEEE Communications Magazine, Vol.49, Issue 6, pp. 89-95, June 2011.

[31] M. Y. Umair, D. Xiao, Y. Dongkai, F. Fanny, "Identification of Interferers in Het-Net in LTE-A systems Based on FeICIC with Cell Range Expansion", IEEE International Conference of Information and Communication Technology (ICoICT), pp. 198-201, 2013.

[32] Guangxiang Yuan, Xiang Zhang and Wenbo Wang, Yang Yang, "Carrier Aggregation for LTE-Advanced Mobile Communication Systems", IEEE Communication Magazine, Vol.48 Issue 2, pp.8893, 2010.

[33] Vikram Chandrasekhar, Jeffrey G. Andrews, "Femtocell Networks: A Survey", IEEE Communications Magazine, Vol. 46, Issue 9, pp. 59-67, September 2008.

[34] V. Chandrasekhar, J. G. Andrews, "Uplink Capacity and Interference Avoidance for Two-Tier Femtocell Networks", IEEE Transactions on Wireless Communications, Vol. 8, Issue 7, pp. 3498-3509, February 2008.

[35] H. Claussen, F. Pivit, "Femtocell Coverage Optimization using Switched Multielement Antennas", IEEE International Conference on Communications (ICC) Dresden, Germany, pp. 1-6, 14-18 June 2009.

[36] Takehiro Nakamura, Satoshi Nagata, Anass Benjebbour, Yoshihisa Kishiyama, "Trends in Small Cell Enhancements in LTE Advanced", IEEE Communication Magazine, vol. 51, Issue 2, 2013.

[37] White paper, "LTE Advanced: Implementing Carrier Aggregation (CA) for Maximizing bandwidth", published by Aricent.

[38] G. de la Roche, A Ladányi, D. Lopez-Perez, C. C. Chong, J. Zhang, "Self-organization for LTE enterprise femtocells", IEEE GLOBECOM Workshops (GC Wkshps), pp. 674-678, 6-10 Dec. 2010.

[39] S. Rangan, "Femto-macro cellular interference control with subband scheduling and interference cancelation", IEEE Globecomm workshop, Miami, FL, pp. 695-700, Dec. 2010.

[40] S. P. Weber, J. G. Andrews, X. Yang, G. de Veciana, "Transmission Capacity of wireless Ad Hoc Networks with Successive Interference Cancellation", IEEE Transaction on Information Theory, Vol. 53, Issue 8, pp. 2799-2814, 2007.

[41] Poongup Lee, Taeyoung Lee, Jangkeun Jeong, and Jitae Shin , "Interference Management in LTE Femtocell Systems Using Fractional Frequency Reuse", IEEE 12th International Conference on Advanced Communication Technology (ICACT), Vol. 1, pp. 1047-1051, Feb.7-10, 2010.

[42] Sternad, M., Ottosson, T., Ahlen, A., Svensson, A., "Attaining both coverage and high spectral efficiency with adaptive OFDM downlinks", Proc. of IEEE $58^{\text {th }}$ Vehicular Technology Conference, vol. 4, pp. 2486-2490, 6-9 Oct. 2003. 


\section{International Journal of Advanced Research in Computer and Communication Engineering} ISO 3297:2007 Certified

Vol. 5, Issue 12, December 2016

[43] 3GPP, "Soft Frequency Reuse Scheme for UTRAN LTE", 3rd Generation Partnership Project (3GPP), R1-050507, May 2005.

[44] Bujar Krasniqi, Martin Wrulich, Christoph F, "Network-Load Dependent Partial Frequency Reuse for LTE", IEEE 9th International Symposium on Communications and Information Technology (ISCIT), pp. 672-676, 28-30 Sept. 2009.

[45] Fang, L., Zhang, X., "Optimal Fractional Frequency Reuse in OFDMA Based Wireless Networks", Proceeding in IEEE $4^{\text {th }}$ international conference on wireless communications network and mobile computing, pp.1-4, 12-14 Oct. 2008.

[46] Yiwei Yu, Eryk Dutkiewicz, Xiaojing Huang and Markus Mueck, "Load Distribution Aware Soft Frequency Reuse for Inter-cell Interference Mitigation and Throughput Maximization in LTE Networks", IEEE international conference on communication (ICC) , Kyoto, Japan, pp. 1-6, 5 - 9 June, 2011.

[47] Ebrahim Baktash, Mehdi Rasti and Ekram Hossain, "Resource Allocation for Dynamic Intra-Cell Subcarrier Reuse in Cooperative OFDMA Wireless Networks", IEEE Transactions on Mobile Computing, vol. 14, No. 7, pp. 1475-1480, July 2015.

[48] R. Almesaeed, A. S. Ameen, E. Mellios, A. Doufexi, A. R. Nix, "A proposed 3D extension to the 3GPP/ITU channel model for 800 $\mathrm{MHz}$ and $2.6 \mathrm{GHz}$ bands", Published in IEEE 8th European Conference on Antennas and Propagation (EuCAP), pp. 3039-3043, April 6-11, 2014

[49] A. Ghosh, J. G. Andrews, N. Mangalvedhe, R. Ratasuk, B. Mondal, M. Cudak, E. Visotsky, T. A. Thomas, P. Xia, H. S. Jo, H. S. Dhillon, and T. D. Novlan, "Heterogeneous cellular networks: From theory to practice", IEEE Communication Magazine, Vol. 50, Issue 6, pp. 54-64, Jun. 2012.

[50] A. Damnjanovic, J. Montojo, Y. Wei, T. Ji, T. Luo, M. Vajapeyam, T. Yoo, O. Song, and D. Malladi, "A survey on 3GPP heterogeneous networks", IEEE Wireless Communication, Vol. 18, no. 3 , pp. $10-21$, Jun. 2011

[51] Han Xiao, Zhiyong Feng, "A Novel Fractional Frequency Reuse Architecture and Interference Coordination Scheme for Multi-cell Network", IEEE $71^{\text {st }}$ Vehicular Technology Conference (VTC 2010-spring), pp. 1-5, May 2010.

[52] David Lo Perez, A kos Ladanyi, Alpar Juttner and Jie Zhang, "OFDMA femtocells: A self-organizing approach for frequency assignment", IEEE 20th International Symposium on Personal, Indoor and Mobile Radio Communications, pp. 2202-2207, 2009

[53] G. Boudreau, J. Panicker, N. Guo, R. Chang, N. Wang, S. Vrzic, "Interference Coordination and Cancellation for 4G Networks", IEEE Communication Magazine, Vol. 47, no. 4, pp. 74-81, Apr. 2009.

[54] Parag KulKarni, Woon Hau Chin, Tim Farnham, "Radio resource management consideration for LTE femto cells", ACM SIGCOMM Computer Communication Review, Vol. 40, no. 1, pp. 26-30, Jan. 2010.

[55] Abbas Hatoum, Rami Langar, Nadjib Aitsaadi, Guy Pujolle, "QFCRA: QoS-based OFDMA Femtocell Resource Allocation Algorithm", IEEE International Conference on CommunicationWireless Networks Symposium, pp. 5151-5156, 2012.

[56] Yu-jung chang, Zhifeng Tao, Jinyun Zhang, C. Jay Kuo, “ A graphbased Approch to multi- cell OFDMA Downlink Resource allocation", TR 2008-092, IEEE Global Telecommunications conference (Globecom 2008), pp. 1-6, Dec. 2008.

[57] K Lee, H Lee, DH Cho, "Collaborative resource allocation for selfhealing in self-organizing networks", IEEE international conference on communication (ICC), Kyoto, Japan, pp. 1-5, 2011

[58] J Zhang, Z Zhang, K Wu, A Huang, "Optimal distributed subchannel, rate and power allocation algorithm in OFDM-based twotier femtocell networks", IEEE $71^{\text {st }}$ Vehicular Technology Conference, Taipei, pp.1-5, 2010.

[59] K. Sundaresan, S. Rangarajan, "Efficient resource management in OFDMA femto cells", 10th ACM International Symposium on Mobile Ad-Hoc Network, NewYork, USA, pp. 33-42, 2009.

[60] V. Chandrasekhar, J. G. Andrews, "Spectrum allocation in two-tier networks", IEEE 42 ${ }^{\text {nd }}$ Asilomar Conference on Signals System and Computing, pp. 1583-1587, 2008.

[61] A. Valcarce, D. Lopez-Perez, G. de la Roche, J. Zhang, "Limited access to OFDMA femtocells", IEEE $20^{\text {th }}$ International Symposium on Personal, Indoor and Mobile Radio Communication, pp. 1-5, 2009.

[62] N. Arulselvan, V. Ramachandran, S. Kalyanasundaram, G. Han, "Distributed power control mechanisms for HSDPA femtocells", IEEE $69^{\text {th }}$ Vehicular Technology Conference (Spring- 2009), pp. 1-5, 2009.
63] S. G. Quan, J. Xu, Y. Y. Kim, "Utility-based rate allocation scheme for mobile video streaming over femtocell networks," Journal of Information Processing System, Korea Science, vol. 5, no. 3, pp. 151-158, Sep. 2009.

[64] G. He, M. Debbah, E. Altman, "Game-theoretic techniques for intelligent wireless networks", International conference on Cognitive systems with Interactive Sensors (COGIS-09) France, Version 1, pp. 1-5, 2009.

[65] S. A. Mahmud, G. M. Khan, H. Zafar, K. Ahmad and N Behttani, "A survey on femtocells: Benefits Deployment Models and Proposed Solutions", IEEE Journal of applied research and technology, Vol. 11, pp. 739-754, 2013.

66] Ana Galindo-Serrano, Lorenza Giupponi, "Self-organized femtocells: a Fuzzy Q-Learning approach", IEEE Wireless Networks, Vol. 20, Issue 3, pp. 441-455, 2014

67] A. Aguilar-Garciaa, S. Fortesa, Mariano Molina-Garcíab, Jaime Calle-Sánchezb, JoséI. Alonsob, Aaron Garridoc, Alfonso Fernández-Duránc, R. Barco, "Location-aware self-organizing methods in femtocell networks", IEEE Computer Networks, Vol. 93, Issue 1, pp. 125-140, 2015.

[68] H. S. Jo, C. Mun, J. Moon, J. G. Yook, "Self-Optimized Coverage Coordination in Femtocell Networks", IEEE Transactions on Wireless Communications, Vol. 9, Issue 10, pp. 2977-2982, 2010.

[69] Yutao Sui , Ismail Guvenc, Tommy Svensson, "Interference management for moving networks in ultra-dense urban scenarios", EURASIP Journal on Wireless Communications and Networking, Vol. 111, pp. 1-32, 2015.

[70] Wei Zheng, Haijun Zhang, Xiaoli Chu, Xiangming Wen, "Mobility robustness optimization in self-organizing LTE femtocell networks", EURASIP Journal on Wireless Communications and Networking, Vol. 23, pp. 1-10, 2013.

[71] Adnan Shahid, Saleem Aslam, Sarmad Sohaib, Hyung Seok Kim, Kyung-Geun Lee, "A self-organized metaheuristic approach towards inter-cell interference management for LTE-Advanced", EURASIP Journal on Wireless Communications and Networking, Vol.-171, pp. 1-15, 2014

[72] Na Chen , Bo Rong, Abdel Mouaki, Wei Li, "Self-Organizing Scheme Based on NFV and SDN Architecture for Future Heterogeneous Networks", IEEE Mobile Networks and Applications, Vol. 20, Issue 4, pp. 466-472, 2015.

[73] M. A. L. Sarker, Moon Ho Lee, "Interference cancellation with an achievable angular channel model based on Coordinate Multi-Point, International Symposium on Communications and Information Technologies (ISCIT), pp. 497 - 501, 2012.

[74] A. Davydov, G. Morozov, I. Bolotin, A. Papathanassiou, "Evaluation of Joint Transmission CoMP in C-RAN based LTE-A HetNets with large coordination areas", IEEE Globecom Workshops (GC Wkshps), pp. 801 - 806, 2013.

[75] Q. Wang, J. Jin, G. Liu, S. Cui, "Coordinated multi-cell beamforming for TD-LTE-advanced systems", IEEE Forty Fifth Asilomar Conference on Signals, Systems and Computers (ASILOMAR), pp. 1304-1308, 6-9 Nov. 2011.

76] Y. Wang, Y. Hu, "Distributed CoMP Transmission For Cell Range Expansion With Almost Blank Subframe In Downlink Heterogeneous Networks", International Conference on Intelligent Computing and Internet of Things (ICIT), pp. 127-130, 17-18 Jan. 2015

[77] S. Xu, J. Han, T. Chen, "Enhanced Inter-Cell Interference Coordination in Heterogeneous Networks for LTE-Advanced", IEEE 75th Vehicular Technology Conference (VTC Spring), pp. 15, 6-9 May 2012

[78] Ian F. Akyildiz , David M. Gutierrez-Estevez, Elias Chavarria Reyes, "The evolution to 4G cellular systems: LTE-Advanced", ELSEVIER journal of Physical Communication, Vol.3, pp. 217244, 2010.

[79] Octavian Fratu, Alexandru Vulpe, Razvan Craciunescu and Simona Halunga, "Small Cell in cellular networks: Challenges of future HetNets", Springer wireless press communication, Vol. 68, Issue 3, pp. 1613-1627, 2014.

[80] Giovanni Nardini, Giovanni Stea, Antonio Virdis and Dario Sabella, "Practical Large-Scale Coordinated Scheduling in LTE-Advanced networks", Springer wireless network, Vol. 22, no. 1, pp. 11-33, Jan. 2016. 